\title{
CONJURAR EL ACCIDENTE DE NACER Migración, población, nacionalidad y ciudadanía
}

\section{Sergio Caggiano}

(iD) https://orcid.org/0000-0003-2718-4391

Centro de Investigaciones Sociales (CIS) del Consejo Nacional de Investigaciones Científicas y Técnicas (CONICET) e Instituto de Desarrollo Económico y Social (IDES), Buenos Aires, Argentina. E-mail: sergio.caggiano@gmail.com

DOI: $10.1590 / 3410109 / 2019$

En términos de pertenencia sociopolítica, el nacimiento de una persona tiene mucho de accidente, pero se trata de un accidente que no sobrevive un instante como tal. Algunos dispositivos sobrevienen a él y otros incluso se le anticipan, interpretándolo y encuadrándolo para que pierda su carácter contingente. Ya codificado, el evento pasa a formar parte de la vida social. Todos aprendemos con naturalidad si vale la paradoja - que somos "naturales" de algún país. La ficción no se sostiene solamente en la mitología nacional y en las razones y pasiones que experimentamos desde la temprana infancia. Se sostiene incluso antes en instituciones y categorías que los Estados ofrecen e imponen como primer marco formal dentro del cual actuar en sociedad. La atribución y adquisición de nacionalidad y ciudadanía se cuentan entre estos primeros dispositivos de regulación.

Artigo recebido em: 29/12/2017

Aprovado em: 11/04/2019
En este marco, las dependencias estatales intervienen respecto de los hijos y las hijas de migrantes desde el nacimiento y aun desde antes de que el nacimiento ocurra. Es una de las formas en que los Estados participan de las implicaciones sociales, jurídicas y políticas de la procreación. Estas intervenciones afectan las nociones y las leyes de nacionalidad y ciudadanía, que tuvieron que ver desde los inicios de los Estados modernos con la regulación del movimiento de personas a través de las fronteras (Stolcke, 2000) y que se han visto problematizadas desde entonces por los movimientos migratorios (Stolcke, 1999; Joppke, 2008; Vertovec, 2006; Escobar, 2007; Pedone y Gil Araújo, 2008; Dumbrava, 2015).

Algunos trabajos de Foucault (2006 y 2007) ofrecen pistas para intentar comprender las dinámicas estatales contemporáneas frente a los flujos migratorios transnacionales. ${ }^{1}$ Conserva gran poder heurístico, en primer lugar, su propuesta de pensar 
la producción de población como uno de los procedimientos constitutivos de los Estados modernos. La producción de población, en su perspectiva, va de la mano de la circulación (de personas, bienes, símbolos y conocimientos) y tiene un lugar central en la biopolítica como tecnología de poder surgida en el siglo XVIII. Si hasta la edad clásica lo crucial era la conquista y el control o conservación de un territorio, a partir de entonces lo será la seguridad de la población y su circulación (Foucault, 2006 y 2007).

Resulta fecunda también la idea foucaultiana del Estado como "una manera de gobernar $[\ldots]$ una manera de hacer" (Foucault, 2006, pp. 291 y 324). No hay aquí un núcleo último de "el" Estado que organice su sentido (su significado y su dirección), sino que el Estado es producido permanentemente como resultado de unas prácticas. De hecho, las dependencias que las llevan adelante pueden estar en conflicto o divergir en sus derroteros. Y estas prácticas y acciones se dan, además, en interacción con organismos internacionales, transnacionales y globales, organizaciones supraestatales o interestatales y organizaciones no gubernamentales de diversa escala de actuación (Dean, 1999).

Estas prácticas y maneras de hacer tienen por fin a la población, crean o producen la población como objeto de su intervención, al tiempo que confirman al Estado como la entidad que se hace cargo de tal regulación. En otras palabras, la producción y reproducción de la población y del Estado se dan de manera entrelazada, como parte de un mismo proceso. Los debates que las migraciones internacionales traen alrededor de la nacionalidad y la ciudadanía exponen esta dinámica constitutiva común de la población y del Estado, que muchas veces tiende a ser opacada por la reificación de ambas entidades.

Este artículo se detiene en dos situaciones que permiten ver las acciones de organismos de cuatro Estados (tres nacionales y uno provincial) en torno a estos asuntos. Las dos situaciones involucran a migrantes procedentes de Bolivia, en un caso residentes en Argentina y en otro en España. La primera sucede en la provincia de Jujuy, limítrofe con Bolivia en el norte argentino, donde algunas agencias del Estado provincial ofrecen resistencia a medidas legislativas impulsadas por el gobierno central, calificándolas como "demasiado permisivas" con los inmigrantes. Según la denuncia de un sector de la iglesia católica, estas agencias obstaculizan el cumplimiento de una ley nacional destinada a facilitar la obtención del Documento Nacional de Identidad (DNI) en el caso de los hijos de bolivianos.

La segunda de las situaciones se desarrolla alrededor de una intervención del Estado Plurinacional de Bolivia (EPB) en el período de gobierno del Movimiento al Socialismo (MAS) y de las reacciones de dependencias estatales españolas. Entre las acciones y políticas de vinculación del EPB con sus "residentes en el exterior", se sancionó la Nueva Constitución Política del Estado (NCPE), que supuso, entre otros cambios sustantivos, uno atinente al régimen de atribución de nacionalidad. Esta modificación y la rápida adecuación de parte de organismos estatales españoles obstruyeron el camino para obtener la nacionalidad española que habían recorrido hasta entonces los migrantes bolivianos para sus hijos nacidos en España.

Los datos empíricos fueron producidos mediante trabajo de campo etnográfico multisituado, revisión de fuentes secundarias y de documentos institucionales, en el marco de un proyecto de largo aliento sobre migraciones, ejercicio de derechos y ciudadanía. El trabajo de campo consistió en visitas, participación en actividades y realización de entrevistas no estructuradas individuales y grupales con migrantes y familiares de migrantes, miembros de oficinas de gobierno y de asociaciones no gubernamentales dedicadas a temas migratorios en Bolivia, Argentina y Espańa durante diferentes etapas entre 2005 y 2015. ${ }^{2}$

Con base en los casos, el artículo procura mostrar formas específicas de producción de población. Estos procesos no son simples, unidireccionales ni exentos de fricciones. Se apreciarán variaciones en el tiempo (los Estados modifican sus normativas y reglamentaciones) y tensiones entre diferentes Estados y niveles de Estado, que en su accionar concreto se entrelazan, además, con organismos y agencias no gubernamentales. Por otro lado, la producción de población no consiste simplemente en la circunscripción de un conjunto social como ciudadanos de un Estado. Las maniobras y medidas concretas de los agentes estatales permitirán ver cómo 
el proceso está desde un comienzo estructurado por valores de clase y de género.

El tratamiento de los casos tiene implicaciones teóricas en torno de la posible emancipación de la ciudadanía respecto de la nacionalidad (Soysal, 1994; Sassen, 2000 y 2002; Bosniak, 2000; Kivisto y Faist, 2007). En relación con ello, veremos mecanismos que van en la dirección inversa, hacia un reforzamiento del vínculo entre ambas nociones. Sin negar que en algunos contextos migratorios contemporáneos el encuentro con una simultaneidad de encuadres institucionales (Levitt y Glick Schiller, 2004), más o menos formalizados, permite a las personas desplegar prácticas ciudadanas alternativas a las consagradas por los Estados, otras prácticas se reconectan a la nacionalidad, como resultado de la capacidad que los Estados conservan y recrean de determinarla positiva o negativamente.

\section{Leyes y papeles nacionales en una provincia de frontera}

Hacia finales de 2003, el Congreso Nacional de Argentina sancionó dos leyes que sacudieron el panorama migratorio del país. Una, de gran trascendencia, fue la Ley de Migraciones 25.871, sancionada en diciembre de aquel año, promulgada en 2004 y reglamentada en 2010, que reconoce la migración como un derecho humano y garantiza a los inmigrantes derechos educativos, sanitarios y laborales sin importar la condición reglamentaria en que se encuentren. Durante esos meses disposiciones complementarias suspendieron las expulsiones de extranjeros en situación documentaria irregular y facilitaron la obtención de residencias temporarias por dos años. La Ley de Migraciones fue celebrada por organizaciones defensoras de los derechos de los migrantes y no despertó, en general, una oposición importante ni recusaciones públicas, sino que fue reconocida como un avance político y administrativo. $^{3}$

En Jujuy la sanción de la ley migratoria tuvo un impacto diferente. Jujuy es una de las provincias más pobres de Argentina, caracterizada por una "crónica situación de retraso" (Lagos y Gutiérrez, 2009, p. 83). Las políticas neoliberales introduci- das en la década de 1970 y que tomarían nuevos bríos en la del noventa afectaron negativamente al conjunto del país, pero "más críticamente a las regiones periféricas" (Idem, ibidem). La quiebra de las economías regionales, particularmente la del noroeste, acrecentó las desigualdades históricas con el área pampeana y el puerto de Buenos Aires. Por otra parte, los actuales noroeste argentino y occidente boliviano estuvieron fuertemente integrados económica y socialmente antes de la conquista española y durante la Colonia. Y más tarde "la dinámica migratoria de Jujuy se vinculó [...] a la migración limítrofe, siendo mucho menor la magnitud de las corrientes migratorias de países no limítrofes" (Sala, 2000, p. 338), que tuvieron un impacto mayor en el resto del país, particularmente en la región pampeana, desde fines del siglo XIX hasta mediados del XX. Estas y otras características socioculturales hacen que para muchos argentinos de la región central del país "Jujuy se "confunda" con Bolivia” (Karasik, 2000, p. 155), lo cual repercute en los sentimientos de pertenencia local de vastos sectores de la sociedad jujeña. Estos sentimientos, junto con la marginalización y posición periférica respecto del gobierno central han generado "reclamos de inclusión en el Estado y la nación, de los que los jujeños parecen sentirse simbólica y socialmente excluidos" (Idem, p. 153).

Entre las reacciones a la sanción de la mencionada ley en la provincia, las críticas señalaron sus deficiencias o directamente sus equivocaciones que, desde esta perspectiva, resultaban de la potestad de Buenos Aires - sede del gobierno nacional - de promulgar leyes que afectan a todo el país. En mis entrevistas de campo, trabajadores de las áreas de salud y de educación públicas, así como funcionarios del Poder Ejecutivo provincial de distinto rango y área, coincidían en que la normativa migratoria no contempla las condiciones específicas de Jujuy y de la región, ni los costos económicos y de cobertura social que la migración conllevaría. Los argumentos se ordenaban según un discurso provincialista que alude al centralismo argentino y apela a los sentimientos de exclusión y marginalización provincial. Sobre esa base la posición provincialista enfrenta al gobierno nacional, presentado como defensor de los migrantes por malicia o por ceguera. 
La restante ley nacional (Ley 25.819) había sido sancionada por el Congreso Nacional poco antes, en noviembre de 2003. Promovía y facilitaba por el plazo de un año la inscripción de nacimientos de nińos de hasta diez años de edad que no hubieran sido inscriptos hasta entonces y la adjudicación del correspondiente DNI. Puesto que en Argentina rige el ius soli, la ley involucraba a todo niño nacido en el país, cualesquiera fuesen las nacionalidades de sus padres. Esta norma también tuvo un impacto singular en Jujuy, donde ocasionó enconadas polémicas durante al menos dos ańos.

A comienzos de 2004 la Pastoral Migratoria de Humahuaca elaboró un "Informe de personas indocumentadas" en los departamentos fronterizos con Bolivia. Dicho informe, presentado en mayo de aquel año ante el Defensor del Pueblo de Jujuy, revelaba que más de quinientas personas - alrededor de un 2,5\% del total de habitantes de la región - se encontraban indocumentadas. Denunciaba que el hospital de la ciudad limítrofe de La Quiaca cobraba ilegalmente a las mujeres bolivianas para dar a luz allí, bajo pena de retener el certificado de nacimiento en caso de no efectuarse este pago ${ }^{4}$,que para inscribir a un niño el Registro Civil exigía a madres y padres bolivianos un certificado de ingreso legal al país que la Gendarmería Nacional muy pocas veces entregaba (lo cual, de todos modos, no debería limitar el derecho del niño o la niña) y que el Registro Civil provincial no admitía certificados de pobreza que evitaran pagar el arancel a quienes no poseían medios para hacerlo.

El entonces encargado del Registro Civil de la provincia argumentaba, por su parte, que "de los quinientos chiquitos indocumentados, cuatrocientos cincuenta eran del país vecino" (funcionario del Registro Civil de la Provincia de Jujuy, marzo de $2005^{5}$ ). La pregunta, desde luego, es qué podía querer decir que "eran del país vecino". Podría significar que habían nacido en Bolivia, pero esto parece de difícil o imposible comprobación. Más probablemente quería decir que eran hijos de bolivianos, pero esto no debería haber modificado en nada el ejercicio de su derecho a la inscripción y a la obtención del DNI argentino. Otros funcionarios del Registro Civil, de la Gendarmería Nacional con sede en Jujuy y, menos previsiblemente, de la
Secretaría de Derechos Humanos de la provincia coincidían en que era legal evitar un otorgamiento "indiscriminado" de documentos.

La Pastoral Migratoria llevó su denuncia a Buenos Aires. Además de cobertura mediática a nivel nacional, consiguió elevar el Informe a la sede central del Registro Nacional de las Personas (Renaper), que realizó una auditoría en la delegación Jujuy y se puso en contacto con la Secretaría de Derechos Humanos de la Nación. Finalmente, un decreto del Poder Ejecutivo nacional de diciembre de 2004 prorrogó la ley 25.819 por un año más y eliminó un requisito presente en la versión original que establecía que la persona que solicitaba la inscripción de un niño y sus testigos debían presentar documento nacional de identidad. Se removía así el ítem que originaba el círculo que reproducía la indocumentación. ${ }^{6}$

\section{Partos en la frontera: fantasías y regulación social}

Para justificar las restricciones en la documentación de niños, los funcionarios del Registro Civil y de otros organismos de la provincia, además de mencionar eventuales inscripciones fraudulentas, evocaban una figura de gran pregnancia social, esgrimida en ámbitos públicos y privados en La Quiaca y en San Salvador - la capital de la provincia - como síntesis de los perjuicios que la provincia sufriría de cara a la migración y el cruce fronterizo: "la mujer boliviana embarazada que cruza la frontera para parir del lado argentino"?

Esta figura, delineada en relación con el presunto aprovechamiento de la cobertura social estatal, en un contexto de distribución del presupuesto nacional considerado injusto e insuficiente para la provincia, justificaba la obstaculización de derechos, desde la documentación personal hasta el acceso a servicios sanitarios. La figura movilizaba, además, una serie de alarmas sobre los beneficios que les daría a las mujeres ser madres en territorio argentino. Tener un hijo en Argentina significa, en virtud de la vigencia del ius soli, tener un hijo argentino, y esto implica habilitar una vía cierta y sencilla para que padres y madres obtengan la resi- 
dencia permanente y los derechos de ciudadanía, así como acelerar los tiempos para que accedan a la nacionalidad, una posibilidad poco frecuente en el contexto internacional actual (Bauböck, 2015). Por ello puede decirse que estas prácticas y medidas en Jujuy "intentan limitar a [...] peligrosas productoras de ciudadanos" (Karasik, 2005, p. 198).

Como señalé en el trabajo citado,

[...] la cuestión de la pertenencia y la exclusión nacional resulta clave, no solo porque es su condición de extranjeras lo que se esgrime como argumento para impedir su acceso gratuito a la atención del parto, sino también porque lo que está en juego es precisamente la obtención (o no) del DNI como madres de un hijo argentino. La obtención de la nacionalidad o de la residencia implicaría la consecución de derechos básicos ligados a la ciudadanía formal y ello, a su vez, significaría la conquista de una posición desde la cual poder demandar por más derechos y más recursos (Caggiano, 2007, pp. 98-99).

El nacimiento de un hijo o hija en el país abre la puerta para "que los trabajadores extranjeros tengan los mismos derechos sociales que los argentinos" (Karasik, 2005, p. 209). Las alarmas alrededor de los partos de mujeres bolivianas parecen vinculadas, así, a los intereses de sectores empresariales de Jujuy contratantes de inmigrantes y de parte de la dirigencia política que representa sus intereses. La específica combinación de explotación y exclusión de la estructura social de la región requiere el control restrictivo de las mujeres bolivianas y de sus embarazos porque tener un hijo argentino abrirá caminos para reclamar mejores condiciones de vida (Bauder, 2008). Los intereses de clase se articulan así con la resistencia que las dependencias estatales provinciales ponen a las leyes de nacionalidad impulsadas desde el Estado nacional.

Resultan sugerentes al respecto algunos hallazgos coincidentes de Mallimacci en su trabajo en Ushuahia, Tierra del Fuego, en el extremo sur patagónico, donde "la migración boliviana se convierte en 'problema' cuando los trabajadores (temporarios) se convierten en residentes (permanentes)" (Mallimacci, 2014, p. 21). También aquí, a más de cuatro mil kilómetros de la frontera con Bolivia, se vuelve recurrente la idea según la cual estas mujeres "vienen a parir" a la ciudad o, al menos, a inscribir a sus hijos aquí. Dado que, según la legislación lo$\mathrm{cal}$, tener un/a hijo/a en la provincia es condición para que los no nacionales puedan acceder a tierras o viviendas (Idem), la capacidad de reproducción de estas mujeres supone un peligro, el de producir trabajadores que compitan de manera permanente por los recursos estatales.

Figuras como la de las mujeres que vienen a parir se entrelazan con la obstaculización de la entrega del DNI para plantear un reto al Estado nacional en su facultad de atribuir la nacionalidad a (quienes pasarían a ser) sus ciudadanos. Si bien no se han materializado leyes o programas provinciales que lleguen a oponerse a las disposiciones del Estado central, las trabas toman forma consuetudinariamente en acciones diseminadas en secretarías y direcciones provinciales, en ventanillas de organismos públicos de salud y educación, así como en los que se encargan directamente de la atribución de nacionalidad y ciudadanía, como los registros civiles.

Junto al argumento provincialista contra el centralismo histórico y la asimétrica estructuración de poder a favor de Buenos Aires, toma forma otra controversia, menos exhibible abiertamente, al menos desde dependencias estatales. Como han mostrado otros trabajos, sectores de la sociedad argentina asumen que los hijos e hijas de bolivianos/ as "son bolivianos", más allá de que hayan nacido en territorio argentino y, por consiguiente, sean jurídicamente argentinos/as por ius soli. En esta asunción convergen concepciones racializadas de la nacionalidad y mecanismos de discriminación (Karasik, 2005; Caggiano, 2005 y 2007). Esta creencia supone la idea de nacionalidades engañosas y ofrece las bases para imputar falseamientos y mentiras a las madres que parirían en un territorio incorrecto. Cuando un funcionario estatal asume que una mujer con documentación boliviana o sin documentación no puede haber tenido un hijo argentino parece no poder asumir que de madre boliviana nazca hijo/a argentino/a. En esta perspectiva, el hecho de que una boliviana tenga a su hijo de "este lado" de la frontera constituye en sí mismo una suerte de engaño. 
Por último, cabe señalar que muchos de los argumentos en este conflicto se formulan en clave de la retórica global alrededor de la seguridad de las fronteras, el tráfico de drogas y la trata y tráfico de personas que han colocado en agenda en los últimos años organismos internacionales como OIM, OEA o Unicef, entre otros (Mansur Dias, 2014). Las simulaciones y mentiras que se imputan a madres y padres en la frontera van desde una adulteración de domicilio hasta el contrabando y el camuflaje de droga en el propio cuerpo o en el de los bebés, pasando por el préstamo de nińos para cruzar la frontera. Del otro lado, la Pastoral Migratoria de la Prelatura de Humahuaca también ha desarrollado desde entonces una intensa actividad en torno a estos temas, particularmente el de "la prevención de la trata, tráfico y explotación sexual comercial de niños, niñas y adolescentes" (VVAA, 2010). A mediados de 2000, además, los Estados parte del Mercado Común del Sur (Mercosur), y el argentino en particular, también incorporaban activamente estos códigos (SDH - MDH, 2007).

\section{Producir población nacional en el exterior}

Desde la llegada de Evo Morales y el MAS al poder en 2006, las migraciones internacionales tuvieron una visibilidad sin precedentes en la agenda política de Bolivia, cuyo Estado se había posicionado hasta entonces como "país receptor" de migrantes (Domenech, 2009). Dicha visibilidad tomó una forma precisa. Funcionarios y documentos oficiales fueron sustituyendo la categoría "migrante" por la de "boliviano residente en el exterior". Este cambio de categorías expresa reorientaciones en las políticas de vinculación del Estado con su población en el exterior y extiende su alcance más allá de sus fronteras territoriales.

Decisiones similares de otras administraciones estatales fueron interpretadas en la década de 1990 como "políticas de vinculación" o de "reincorporación transnacional” (Guarnizo y Smith, 1998; Goldring, 2002). A comienzos de este siglo se habían registrado políticas de este tipo en países como México, Colombia, República Dominicana, El Salvador, Guatemala, Filipinas, Eritrea, India, Croacia,
Ecuador, Brasil, Haití y Portugal, y se identificaron como antecedentes los casos de Hungría, Italia, Japón, China, Israel, Turquía y Argelia (Smith, 2003; Glick Schiller, 2005). Para dar cuenta de estos fenómenos, se propusieron conceptos como el de "Estados-nación desterritorializados" (Basch et al., 1994; Solomon, 2009), "Estados-nación transnacionales" (Glick Schiller y Fouron, 1999; Glick Schiller, 2005), "políticas diaspóricas" (Bauböck, 2002; Lafleur, 2012; Van Dongen, 2017) etc.

Entre las medidas de revinculación del EPB (promoción de la doble nacionalidad, facilitación de la documentación de bolivianos y bolivianas fuera del país, reconocimiento del derecho al voto en el exterior, programa de Consulados Móviles), merece especial atención una modificación en el otorgamiento de la nacionalidad instaurado por la Nueva Constitución Política del Estado (NCPE), al establecer en su artículo 141 que "son bolivianas y bolivianos por nacimiento, las personas nacidas en el territorio boliviano [... y y las personas nacidas en el extranjero, de madre boliviana o de padre boliviano". El artículo estipula esta segunda alternativa de manera automática, los hijos de bolivianos en el extranjero serán bolivianos sin mediar trámite previo. En términos jurídicos, se sanciona un régimen mixto de concesión de nacionalidad de origen que hace lugar al ius soli y al ius sanguinis. ${ }^{8}$

La modificación normativa ha impedido que, en adelante, hijos e hijas de bolivianos nacidos en España puedan obtener de manera sencilla su nacionalidad española, porque de acuerdo con el Código Civil de España, dada la vigencia del ius sanguinis, son españoles los hijos de españoles y los hijos de extranjeros reciben, en principio, la nacionalidad de los padres. Por cierto, un ítem de protección de los menores que busca evitar situaciones de apatridia abre la posibilidad de que estos puedan adquirir la nacionalidad española, y eso es lo que habitualmente sucedía con hijos e hijas de bolivianos antes de la promulgación de la nueva Constitución. La normativa boliviana anterior abría para los hijos de bolivianos nacidos en el extranjero la posibilidad de adquirir la nacionalidad si eran registrados por sus padres en la oficina consular correspondiente. Pero con ello se abría también la chance de obtener la nacionalidad espańola: los 
padres no inscribían al niño o la nińa en el Consulado de Bolivia y, por tanto, no era boliviano. A continuación, con el certificado de no inscripción consular se solicitaba la nacionalidad española "con valor de simple presunción”, la cual era concedida de inmediato para evitar la apatridia del niño.

La modificación normativa que trajo la NCPE implica que al no existir ya el requisito de inscripción consular tampoco existe la alternativa de la no inscripción. A partir de ello, los registros civiles de España determinaron que los hijos de bolivianos ya no podrían acogerse a la solicitud de nacionalidad española con valor de simple presunción. Se disponía así hacia el adelante un recorrido escabroso y sin garantía de consecución.

De esta manera, una medida de vinculación del $\mathrm{EPB}$ con los residentes en el exterior, dirigida en principio a garantizar protección y simplificar el acceso a derechos al allanar el camino de obtención de una pertenencia jurídica, coloca simultáneamente obstáculos a que estos mismos residentes puedan realizar el trámite básico y fundamental para reclamar derechos y protección en Espańa y en Europa, y acaba siendo, paradójicamente, compatible con el endurecimiento de las políticas migratorias en esta región.

Muchos de los bolivianos que advirtieron cómo podría afectarlos el nuevo escenario manifestaron su sorpresa y su rechazo. Hubo residentes en Madrid, por ejemplo, que se acercaron perplejos a las oficinas del Defensor del Pueblo de España con intención de profundizar en el tema y responsabilizaron de la nueva situación al Estado español (fuente del Defensor del Pueblo, Madrid, mayo de 2012). Funcionarios del Estado boliviano también reaccionaron. El entonces vicecanciller Hugo Fernández señaló que la nueva Constitución había sido malinterpretada y que se realizarían gestiones con el gobierno español para salvar el "problema de tipo legal que surgió por el cambio de la Constitución”. Por su parte, Waldo Albarracín, ex Defensor del Pueblo de Bolivia, agregó que no se podían "adoptar decisiones en territorio español sobre la base de normas surgidas en otros países para restringir derechos de personas que viven dentro de la jurisdicción española” (Los Tiempos, 2009).

Hubo asimismo medidas concretas de parte del gobierno boliviano, como la emisión consular de certificados de no inscripción de los menores nacidos en España luego de la reforma constitucional, que los padres y madres bolivianos utilizaron como documentación probatoria en los trámites que iniciaron en los registros civiles de España para solicitar la declaración de nacionalidad española con valor de simple presunción para sus hijos. También la Embajada de Bolivia emitió con este propósito notas explicativas que indicaban, por ejemplo, que la NCPE "no contiene un mandato tácito" de nacionalidad y que reconoce "el derecho a la doble nacionalidad y la discrecionalidad de los padres para su ejercicio" (Ministerio de Justicia de Espańa, 2012, p. 20).

La Dirección General de los Registros y del Notariado de España trató una cantidad de estas solicitudes desde 2009. El criterio de esta Dirección General es taxativo: se acepta que son españoles iure soli los nacidos en España de padres bolivianos antes de la entrada en vigor de la NCPE y se establece que no lo son los nacidos tras la entrada en vigor de aquella por corresponderles la nacionalidad boliviana de sus padres. Su argumento se apoya en que la Constitución de Bolivia adopta "un régimen de atribución de la nacionalidad ius sanguinis", en que de acuerdo con este régimen los padres no detentan derecho a que la nacionalidad de sus hijos sea o no atribuida y en que la inscripción del niño es una mera formalización de la adquisición (Ministerio de Justicia de España, 2012, p. 20-21).

\section{Lazos estatales y nacionalidad automática}

Medidas como la modificación constitucional alientan un proceso de producción de población (Foucault, 2006) que busca resolver una preocupación oficial que precisamente se explicita en términos de una pérdida de "la capacidad de reproducción de nuestra población" (funcionario de la Dirección General de Asuntos Consulares de Bolivia, marzo de 2012). Resulta clave aquí la automaticidad de la nacionalidad en el extranjero que la NCPE consagra. Al ser automática, la nacionalidad es involuntaria. Es cierto que la atribución de nacionalidad de origen - es decir, dada al nacer - no puede ser nunca voluntaria y es casi siempre automática (Trucco, 2007, p. 3). Pero para los migran- 
tes en España la nacionalidad boliviana automática de sus hijos constituye una novedad que choca contra la posibilidad de obtener la nacionalidad española, tal como sucedía hasta poco tiempo atrás. La automaticidad, de esta manera, obstruye otras posibles vías de nacionalización y, por ello, puede ser algo no deseado y resistido.

Las intervenciones estatales distinguen claramente el territorio de la población, o al menos los énfasis específicos que ellos reciben cada vez. Con las acciones que apuntan a los emigrantes se trata de producir población nacional en el exterior del territorio. El lenguaje jurídico contempla esta distinción al diferenciar la "supremacía personal", originada en el vínculo de nacionalidad y que permite al Estado ejercer sus poderes respecto a quienes considera sus nacionales, aunque ellos se encuentren fuera de su territorio, de la "supremacía territorial”, que el Estado detenta respecto a los extranjeros por el hecho de encontrarse estos "dentro del ámbito en que el Estado ejerce su soberanía territorial" (Idem, p.1).

Con la aplicación del ius sanguinis automático en el extranjero, Bolivia - como otros países - ejerce fuera de su territorio una suerte de supremacía personal sobre los (aún) no nacionales, a quienes nacionaliza en su nacimiento o, en todo caso, prevé la aplicación del ius sanguinis sobre quienes aún no nacieron en el extranjero. El Estado anticipa la producción de una población nacional haciendo navegar su supremacía personal en los flujos de la sangre y la descendencia.

Qué consecuencias y qué reacciones pueda generar un conjunto de medidas del tipo de las impulsadas por un Estado como el boliviano, que coloca en la agenda de gobierno la emigración y propone el "apoyo a la 'vida transnacional"” (MRE y OIM, 2011, p. 21) en un contexto de transformación del Estado y activación de políticas de descolonización y reducción de desigualdades es un interrogante que permanece abierto porque refiere a un proceso social: son hombres y mujeres haciendo su vida quienes se amoldarán o no a la nueva situación. Las consecuencias de las políticas de nacionalización a distancia no pueden pensarse monolíticamente, además, porque alcanzan a poblaciones que se rigen también por los encuadres jurídicos específicos de sus respectivas sociedades de residencia. De este modo, por ejemplo, al mismo tiempo que la nacionalidad automática implanta una limitación en España, en un país como Argentina, donde rige el ius soli, supone virtualmente la doble nacionalidad automática (y la doble ciudadanía), lo cual puede traer aparejadas ventajas relativas.

\section{Producción de población, dependencias estatales y múltiples relaciones de poder}

Como algunos autores inspirados en Foucault han planteado, es útil pensar la gubernamentalidad a nivel del sistema de Estados, lo que implica pensar este sistema como un régimen de gobierno que opera sin un único centro de control (Dean, 1999 y 2007). Este sistema separa poblaciones y distribuye los derechos y responsabilidades con los que los Estados regulan sus entradas y salidas. En esta línea, puede considerarse a la ciudadanía no solamente desde una perspectiva interna al Estado, como un desarrollo progresivo de capacidades, derechos y responsabilidades en su interior, sino desde su lado externo, desde donde puede verse que ella "funciona como un marcador de identificación, informando a las autoridades sobre la pertenencia o no pertenencia de individuos particulares" (Kalm, 2008 , p. $103^{9}$ ). Se vuelve crucial, entonces, establecer la diferencia entre quién es quién, a dónde pertenece cada quien y dónde puede o le corresponde estar. Entre las tecnologías que posibilitaron esto, además de los censos y otros sistemas de registro, ha sido crucial la invención de los documentos de identidad y, para la movilidad internacional, la del moderno sistema de pasaportes (Torpey, 2000).

Como analiza Kalm (2008), los organismos internacionales establecen lineamientos claros para orientar acciones estatales en la gestión de las migraciones. Por un lado, promueven la cooperación de los Estados con un amplio abanico de actores, entre los que se cuentan organizaciones no gubernamentales de distinto alcance, organismos regionales interestatales (como el Mercosur o la Unión Europea), asociaciones del sector privado, otros Estados, así como los propios organismos que redactan estas recomendaciones, como las Naciones Unidas y al- 
gunas de sus agencias (ACNUR - Alto Comisionado de las Naciones Unidas para los Refugiados, UNFPA - Fondo de Población de las Naciones Unidas, OIT - Organización Internacional del Trabajo, OIM - Organización Internacional para las Migraciones y otras) o el Global Migration Group, que reúne a varias de las anteriores con la Oficina de Drogas y Crimen de las Naciones Unidas y el Banco Mundial. Por otro, insisten en convalidar una de las principales prerrogativas reconocidas a cada Estado nación, que es la de controlar los flujos de personas, la salida y la entrada de/a su territorio, es decir, el derecho esencial de los Estados modernos a ejercer "el monopolio de los medios legítimos de movimiento" (Torpey, 2000, pp. 4 y ss.).

¿Qué muestran nuestros casos a propósito de la complexión de las agencias que llevan adelante las acciones estatales, consolidando a los Estados mismos en su accionar? En primer lugar, son agencias que participan de conflictos. Está claro, como en el caso desatado por la nueva Constitución boliviana, que hay tensiones entre dependencias estatales nacionales de distintos países, bolivianas de un lado y españolas de otro. Pero también hay conflictos entre niveles de Estado, como muestra el primer caso entre leyes del Congreso Nacional argentino y el Registro Civil de Jujuy. Además del sistema de gobierno federal que rige en Argentina, en esta coyuntura los diálogos y la cooperación con organismos supranacionales y organizaciones no gubernamentales globales ofrecen a las dependencias estatales provinciales vías por las cuales desafiar al Estado central en prerrogativas como la de otorgar documentos de nacionalidad. Por último, hay también conflictos dentro del mismo nivel de un mismo Estado, como en la competencia por montos de cooperación o cuotas de presupuesto entre, por ejemplo, la Dirección General de Asuntos Consulares, dependiente del Ministerio de Relaciones Exteriores de Bolivia, y el Servicio Nacional de Migraciones (Senamig), dependiente del Ministerio de Gobierno (Domenech, 2009).

De la misma forma que los conflictos, también tienen lugar a distinta escala las alianzas y la coordinación de acciones, que pueden darse entre dependencias gubernamentales locales, como el Registro Civil de Jujuy y la Secretaría de Derechos Humanos provincial, o entre ellas y dependencias de nivel nacional, como la Gendarmería. Las alianzas y coordinaciones, por lo demás, como sugirieran algunos organismos internacionales, van más allá de los entes gubernamentales. Todos ellos se conectan con algunos de estos organismos internacionales, así como con organizaciones no gubernamentales nacionales y extranjeras, que brindan apoyo financiero o técnico, encuadres conceptuales, herramientas jurídicas y administrativas, y a veces protagonizan el conflicto, como sucede en el primer caso con una sección regional de la iglesia católica. Las dependencias estatales nacionales, como se dijo, actúan también dentro de unidades políticas más amplias, como Argentina y Bolivia respecto del Mercosur o Espańa respecto de la Unión Europea. A la hora de diseñar políticas de cara a los "residentes en el exterior", por último, las partes bolivianas no pueden ignorar sus relaciones históricas con aquellos países ni las relaciones de fuerza entre ellos, o sea, especialmente de cara a sus contrapartes españolas, su posición vulnerable como aparatos de Estado de un país de emisión en este régimen de regulación global (Glick Schiller, 2007).

El Estado que motoriza las situaciones analizadas o comparece en ellas está constituido inarmónicamente por estas agencias concretas que, más allá de actuar en estructuras institucionales jerárquicas y tener historias en común, operan también con una autonomía a partir de la que configuran sus múltiples relaciones. Las maneras de hacer que constituyen a los Estados se forjan en alianzas y disputas, se despliegan en diálogos multiescalares de los que participan organismos internacionales y globales y organizaciones locales, muchas veces en red. Estas acciones no ordenadas a priori por una fuerza motriz de dirección única predeterminada tienen efectos contundentes sobre la vida de las personas, incluso antes de nacer.

Entre estos efectos puede estar que un niño obtenga o no la nacionalidad argentina o española, y sus padres la residencia o su propia nacionalidad, que la nacionalidad boliviana automática facilite una doble ciudadanía o que, por el contrario, la limite etc. El régimen de poder de la biopolítica produce a la población como su objeto, y su bienestar - o mejor, el mero hacer vivir a esta población - 
supone también producir un espacio exterior, pero constitutivo, de seres humanos prescindibles a los que se puede dejar morir (Foucault, 2007). Niños y niñas sin documentos o con documentos que les impiden acceder a otros documentos, y padres y madres que por ello se ven impedidos de acceder a sus documentos recuerdan que, parafraseando a Joseph Roth, una persona sin papeles vale menos que un papel.

Por otra parte, además de los actores institucionales que, participando de estos diálogos multiescalares, buscan explícitamente regular la producción de población en relación con los flujos migratorios, otros actores sociales y otros valores se entretejen de manera menos manifiesta en este juego. El primero de los casos deja ver algo que está presente en los dos: que los intereses sectoriales, más precisamente de clase, penetran estos procesos. Las resistencias provinciales a las leyes nacionales están permeadas por intereses de clase en la medida en que son algunos sectores empresariales de la provincia los beneficiarios inmediatos de mantener una masa de posibles empleados en situación de vulnerabilidad documentaria, sin sus derechos ciudadanos garantizados (Bauder, 2008).

Asimismo, la recurrencia descalificatoria de la imagen de "la mujer boliviana que cruza la frontera para parir del lado argentino", reiterada por funcionarios estatales en la provincia de Jujuy y en geografías tan distantes como el sur de la Patagonia (Mallimacci, 2014), recuerda que, como analistas feministas observaron hace tiempo, la producción y el sostenimiento de la desigualdad en las sociedades de clase requiere un sistema ideológico e institucional patriarcal y heteronormativo que promueva el control de las mujeres, de sus cuerpos y su capacidad reproductiva (Stolcke, 1992; Davin, 1997).

En síntesis, los desplazamientos poblacionales contemporáneos actualizan uno de los dilemas primordiales y constitutivos del período formativo de los Estados-nación modernos: determinar las condiciones para establecer la pertenencia a un Estado, "circunscribir la colectividad de ciudadanos" (Stolcke, 2000, p. 23; Brubaker, 2010). Ahora bien, lo que los casos estudiados permiten apreciar no es simplemente la determinación positiva de un conjunto de personas como población de un Es- tado. Son procesos tensos y disputados en los que las agencias estatales producen población y se legitiman a sí mismas en interacciones no exentas de conflicto con otras agencias estatales y no estatales a diferente escala (Sikkink, 2003). Y además, la producción de población que hace foco en los hijos e hijas de migrantes está siempre ya cargada de intereses de clase y de género, y corrobora y anticipa desigualdades que complementan las que instaura el reconocimiento o el desconocimiento de la pertenencia nacional y ciudadana.

\section{La persistencia de la nacionalidad en las ciudadanías contemporáneas}

Las cuestiones que se desatan alrededor de las migraciones, los Estados, la globalización y el transnacionalismo abren la discusión en torno de la relación entre nacionalidad y ciudadanía (Smith y Guarnizo, 2009), comúnmente asumida como de superposición o de subsunción de ambas en un único estatus indistinto. Incluso en términos legales ambos términos son a menudo usados como sinónimos. ${ }^{10}$ Dicha subsunción de "nacionalidad" y "ciudadanía" en un único estatus se vincula con la dificultad para distinguir los requerimientos formales para adquirir la ciudadanía (la nacionalidad) de los derechos sustantivos que ella implica o "las condiciones para ser miembro de un estado nacional y los derechos derivados de serlo" (Stolcke, 2000, p. 24; Brubaker, 1992; Jones-Correa, 2001). Además, aunque técnicamente cada término refleja una estructura legal diferente - la ciudadanía se limita a la dimensión nacional mientras que la nacionalidad refiere a la dimensión legal internacional en el contexto de un sistema interestatal -, ambos se relacionan con el Estado nación, identificando "la condición legal de un individuo en términos de pertenencia a un Estado" (Sassen, 2002, p. 7).

Ahora bien, esta superposición es histórica, no siempre se dio por descontada y, según algunos autores (Sassen, 2002), comienza a no ser tomada así en las últimas décadas - en cierta medida como consecuencia de la movilidad humana contemporánea -, cuando emergen formas de emancipación de la ciudadanía respecto de la nacionalidad (Kivisto 
y Faist, 2007). En un contexto global en que los Estados compiten con numerosos ámbitos alternativos en la definición de derechos, obligaciones, afiliaciones e identidades se ha puesto en discusión el carácter presuntamente intrínseco de la relación entre la ciudadanía y el Estado, y se han señalado formas postnacionales, transnacionales o denacionales de ciudadanía (Soysal, 1994; Besserer, 1999; Bosniak, 2000; Sassen, 2000 y 2002; Liebert, 2005; Smith, 2007; Bloemraad et al., 2008; Smith y Bakker, 2008; Calderón Chelius, 2013).

Sin negar la existencia de estas formas alternativas de ciudadanía, intervenciones estatales como las analizadas aquí estrechan la relación entre ciudadanía y nacionalidad. En el caso de la nacionalidad argentina para los hijos de padres bolivianos, ella no solo impacta directamente en las prácticas ciudadanas futuras de estos niños, sino en las de sus padres, en terrenos sensibles como el laboral y el del acceso a programas sociales. En el caso de la modificación que supuso la NCPE, la nacionalidad boliviana muestra su carácter constitutivo para el ejercicio de la ciudadanía en Bolivia, al tiempo que la producción automática de bolivianos en el extranjero obstaculizará a algunos migrantes el despliegue de tácticas de ciudadanización en España. No se trata solo de insistir en lo que ya otros autores abundaron: la importancia de los papeles en el ejercicio de derechos, sino de advertir acerca de estas precisas diligencias estatales alrededor de las migraciones y la movilidad humana y acerca de su impacto en las prácticas ciudadanas y el ejercicio de derechos de los actuales - y de los futuros - (no) ciudadanos. Las agencias estatales se muestran muy activas en la dinámica de desajustes y reajustes de los vínculos entre nacionalidad y ciudadanía (Brubaker, 2010).

Complementariamente, estas diligencias estatales encuadran la interlocución con las personas de manera de hacer que algunas de sus propias prácticas contribuyan a este estrechamiento. En momentos críticos en que un hijo puede quedar sin reconocimiento de parte de Estado alguno o en que unos determinados derechos ciudadanos nacionalmente definidos pueden serle a uno arrebatados, las acciones de los migrantes parecen reforzar también de manera buscada o no - la relación entre ciuda- danía y nacionalidad. La observación de Sassen de que "la ciudadanía - aun situada en un marco institucional «nacional» - es una institución que puede cambiar del mismo modo en que ha cambiado el significado de lo 'nacional' (Sassen, 2002, p. 16) debería permitirnos apreciar que los desplazamientos entre nacionalidad y ciudadanía se dan en direcciones variadas $\mathrm{o}$, al menos, en dos direcciones: de alejamiento pero también de acercamiento.

\section{Conclusiones}

Dos hermanas de una familia del sur de Bolivia deciden emigrar a comienzos de 2000, una por tierra hacia el norte argentino, otra por aire hacia Madrid. Pasado el tiempo, esta última tiene dos hijos en España y aquella uno en Argentina. El que nació en este país es argentino porque rige el ius soli y porque el Estado argentino lo promueve, aunque dependencias del Estado jujeño obstaculicen fuertemente esa atribución de nacionalidad. El primero que nació en España, en 2007, es ciudadano español y comunitario porque sus padres pudieron solicitar la nacionalidad con valor de simple presunción para evitar la apatridia. El segundo, nacido tras la puesta en vigor de la NCPE, es boliviano, en virtud de la automaticidad del ius sanguinis que ella consagra. Podríamos agregar imaginariamente el niño de una tercera hermana que, nacido en los Estados Unidos, sea ciudadano de ese país, pero sea pasible de ser deportado junto con sus padres sin papeles. De manera condensada, la vida de estas personas muestra cómo sus desplazamientos espaciales y, sobre todo, las intervenciones estatales que les conciernen transforman profundamente posiciones que en un comienzo estaban tan emparentadas.

La situación expone dos puntos con claridad. El primero es la variabilidad temporal y espacial de estos procesos. Temporal porque las modificaciones reglamentarias pueden darse en el lapso de un puñado de años, y espacial porque las diferencias son más o menos formalizadas en intervenciones de agencias estatales de distinto nivel y jurisdicción. El segundo punto es que la potestad que los Estados detentan para definir quién es ciudadano y quién migrante, y quién migrante de qué país y en 
qué condiciones, es fundamental en el control del proyecto migratorio y de vida. Se definen las condiciones para estar dentro y fuera y para establecer las formas de la pertenencia.

Ese hecho "natural" que es el nacimiento de un niño, vivido en las sociedades occidentales principalmente como acontecimiento íntimo y familiar, se revela locus privilegiado de la acción de conformación permanente del Estado, a través de prácticas por las que se da a sí mismo sentido y existencia. Cualquier política migratoria convalida el hecho de que hay o habrá unos (los insiders) cuyas voces y reclamos valen y otros (los outsiders) cuya legitimidad podrá al menos cuestionarse (Kalm, 2008). Las políticas positivas y negativas de asignación de nacionalidad y ciudadanía son, entonces, parte constitutiva de los procesos de producción de población, y las dependencias estatales se re-crean al modificar y renovar las acciones que les dan forma.

En el contexto social actual de movilidades espaciales, crecen las acciones e intervenciones sobre poblaciones en movimiento, actualizándose uno de los rasgos que caracterizan a los Estados desde el siglo XVIII, cuando empiezan a definirse crecientemente, además de por su territorio, por "la masa de la población, con su volumen [y] su densidad" y por las dinámicas de circulación que la afectan (Foucault, 2006, p. 137). Los flujos en general, y los flujos poblacionales en particular, caracterizan el momento actual y son uno de los elementos que especifican las intervenciones estatales contemporáneas. Otro es la articulación de las agencias estatales con organismos internacionales y con organizaciones no gubernamentales de carácter transnacional o global, que tienen gran incidencia no ya en permitir o impedir la circulación, sino en definir el flujo, en “codificarlo” (Deleuze y Guattari, 1974), en instalar las categorías con las cuales interpretarlo y actuar sobre él, canalizarlo u ordenarlo.

En los casos analizados, diferentes agentes estatales de distintos países y niveles participan formulando e interpretando normas para la promoción u obstrucción de derechos de los migrantes, fundamentalmente en clave de sangre y descendencia o de territorio (que, ciertamente, no son equivalentes ni en sus efectos inmediatos ni en las condiciones que colocan para la acción de los propios migran- tes). Con estas intervenciones las dependencias estatales estrechan la relación entre nacionalidad y ciudadanía, al recordar el carácter fundante que puede adquirir en estos momentos críticos la pertenencia formal a una unidad política como requisito para determinadas prácticas ciudadanas.

$\mathrm{Al}$ estrechar este vínculo, las propias dependencias estatales fortifican sus posiciones relativas, al tiempo que permean intereses y valores de clase y de género, entre otros. Se estructuran desigualdades entre quienes quedan dentro y quienes quedan fuera, y además esa división es simple solo en apariencia, ya que las modalidades específicas en que se instaura la nacionalidad y la ciudadanía consagran también asimetrías y jerarquías entre quienes quedan dentro. Recurriendo a la figura de Shachar (2009), en la "lotería del derecho por nacimiento" (birth right lottery), y palmariamente en la del derecho por nacimiento en contextos migratorios, se juega con cartones adulterados por actores con cuotas dispares de poder.

Como en todo proceso social, hay ataduras y también resistencias, o intentos de desembarazarse de esas ataduras. En las "constelaciones de ciudadanía” (Bauböck, 2010) los migrantes actúan y pueden muy bien lograr sus objetivos al disponer estrategias para el acceso a derechos que presumen las más eficaces de acuerdo con la "estructura de oportunidades ciudadanas" (Bauböck, 2010, p. 849) y el "sistema de preferencias" particular del Estado de residencia en cuestión (Mateos y Durand, 2012, p. 17). Pero en este escenario las ataduras son fuertes y el margen de maniobra de los migrantes limitado. Si se consideran los impedimentos de padres y madres bolivianos en Jujuy o en España para inscribir a sus hijos, la lucha se devela extremadamente difícil. Y esto sin siquiera intentar mirar el fenómeno desde la perspectiva de los niños y las niñas.

Las intervenciones estatales no son absolutas ni definitivas, pero son contundentes en cuanto al control del proyecto migratorio. Mediante el recurso a herramientas clásicas, como la atribución de nacionalidad, las dependencias estatales habilitan $\mathrm{u}$ obstruyen prácticas ciudadanas en su territorio y en otros. Si bien los migrantes desplegarán prácticas ciudadanas no necesariamente consagradas institucionalmente y podrán obtener reconocimientos de 
otros actores sociales y políticos, en muchas de las ocasiones en que se definan y redefinan sus posiciones y relaciones en torno al trabajo, la participación política, la definición misma de necesidades y derechos, estas tempranas intervenciones estatales multiescalares y articuladas a las de otros actores globales y transnacionales volverán a mostrar su peso y estarán allí ordenando una parte sustantiva del tablero donde el juego se desarrollará.

\section{Notas}

1 Sobre los usos posibles de Foucault en los estudios migratorios, Walters (2015).

2 Las diferentes etapas de trabajo de campo fueron realizadas como becario de posgrado y luego como investigador del Consejo Nacional de Investigaciones Científicas y Técnicas (Conicet), gracias al apoyo recibido como responsable de un Proyecto de Investigación - PICT Joven de la Agencia Nacional de Promoción Científica y Técnica (FONCyT - ANPCyT), 2011 - 2013, y como becario posdoctoral de la red desiguALdades.net (Instituto Latinoamericano - Universidad Libre e Instituto Iberoamericano de Berlín), 2012 - 2013. Fueron realizadas 39 entrevistas en La Paz y El Alto (Bolivia), 31 en Buenos Aires y 27 en Jujuy (Argentina) y 27 en Madrid (España).

3 No obstante, sectores políticos de derecha en el gobierno de Buenos Aires desde 2007 y en el nacional desde 2015 han promovido la creciente criminalización de las migraciones y la intensificación de los controles fronterizos (Caggiano, 2017).

4 Los directivos del hospital negaron el hecho y el Defensor del Pueblo recomendó investigar la denuncia. En mi trabajo de campo y el de Karasik (2005) aparecen referencias directas, aunque matizadas, a estos cobros informales.

5 Todas las entrevistas fueron realizadas por el autor.

6 Complementariamente, medidas como el Programa "Patria Grande" (2004/2006) propiciaron la regularización documentaria de los migrantes nativos de países pertenecientes al Mercosur y sus Estados Asociados.

7 En otro artículo analicé en torno de esta figura las ansiedades de género, clase social y raciales disparadas por el cruce de fronteras nacionales (Caggiano, 2007).

8 Recupero algunas líneas del argumento desarrollado en Caggiano (2017).
9 Todas las traducciones son del autor.

10 En rigor, al lado de grandes similitudes, hay una miríada de pequeñas diferencias entre países en la definición de cada concepto y de su relación (ver varios informes en EUDO Citizenship: http://eudo-citizenship.eu; consultado en 06/06/2017).

\section{BIBLIOGRAFÍA}

BASCH, Linda; GLICK SCHILLER, Nina \& SZANTON BLANC, Cristina. (1994), $\mathrm{Na}$ tions unbound: transnational proyects, postcolonial predicaments, and deterritorialized $\mathrm{Na}$ tions-States. Amsterdam, Gordon and Breach Publishers.

BAUBÖCK, Rainer. (2002), “Towards a political theory of migrant transnacionalism". IWE Working Paper Series, 34: 1-17.

BAUBÖCK, Rainer. (2010), "Studying citizenship constellations". Journal of Ethnic \& Migration Studies, 36 (5): 847-859.

BAUBÖCK, Rainer. (2015), "Ius filiationis: a defence of citizenship by descent", in C. Dumbrava \& R. Bauböck (eds.), Bloodlines and belonging: time to abandon ius sanguinis?, EUI Working Paper RSCAS 2015/80.

BAUDER, Harald. (2008), "Citizenship as capital: the distinction of migrant labor". Alternatives: Global, Local, Political, 33 (3): 315-333.

BESSERER, Federico. (1999), "Estudios transnacionales y ciudadanía transnacional”, in G. Mummert (ed.), Fronteras fragmentadas. Zamora, El Colegio de Michoacán/Centro de Investigaciones y Desarrollo del Estado de Michoacán.

BLOEMRAAD, Irene; KORTEWEG, Anna \& YURDAKUL, Gökçe. (2008), "Citizenship and immigration: multiculturalism, assimilation, and challenges to the Nation-State". Annual Review of Sociology, 34: 153-179.

BOSNIAK, Linda. (2000), "Citizenship Denationalized”. Indiana Journal of Global Legal Studies, 7 (2): 447-509.

BRUBAKER, Rogers. (1992), Citizenship and Nationhood in France and Germany. Cambridge, Harvard University Press. 
BRUBAKER, Rogers. (2010), "Migration, =membership, and the modern Nation-State: internal and external dimensions of the politics of belonging". The Journal of Interdisciplinary History, 41(1): 61-78.

CALDERÓN CHELIUS, Leticia. (2013),"Los límites de la ciudadanía clásica: las coordenadas del debate teórico contemporáneo", in C. Amescua et al. (coords.), Politica en movimiento: Estado, ciudadanía, exilio y migración en América Latina, México, Centro Regional de Investigaciones Multidisciplinarias, Unam/ Díaz de Santos.

DAVIN, Anna. (1997), "Imperialism and motherhood", in F. Cooper, y A. L. Stoler (eds.), Tensions of empire: colonial cultures in a bourgeois world, California, University of California Press.

DEAN, Mitchell. (1999), Governmentality: Power and Rule in Modern Society. London, SAGE.

DEAN, Mitchell. (2007), Governing societies: political perspectives on domestic and international rule. Buckingham, Open University Press.

DELEUZE, Gilles \& GUATTARI, Felix. (1974), El antiedipo: capitalismo y esquizofrenia. Buenos Aires, Corregidor.

DOMENECH, Eduardo. (2009), "Avatares de la política migratoria en Bolivia: el Estado y los emigrantes como nacionales en el exterior", in VVAA, Migraciones contemporáneas: contribución al debate, La Paz, CAF, CIDES-UMSA, Plural editores.

DUMBRAVA, Costica. (2015), "Super-foreigners and sub-citizens: mapping ethno-national hierarchies of foreignness and citizenship in Europe". Ethnopolitics, 14 (3): 296-310.

ESCOBAR, Cristina. (2007), "Migración y derechos ciudadanos: el caso mexicano", in A. Portes \& M. Ariza (comps.), El pais transnacional: migración mexicana y cambio social a través de la frontera, México, Universidad Nacional Autónoma de México - Instituto de Investigaciones Sociales.

FOUCAULT, Michel. (2006), Seguridad, territorio, población. Buenos Aires, Fondo de Cultura Económica.
FOUCAULT, Michel. (2007), Nacimiento de la biopolitica. Buenos Aires, Fondo de Cultura Económica.

GLICK SCHILLER, Nina \& FOURON, Georges. (1999), "Terrains of blood and nation: Haitian transnational social fields". Ethnic and Racial Studies, 22 (2): 340-366.

GLICK SCHILLER, Nina. (2005), "Blood and belonging: long-distance nationalism and the world beyond", in S. McKinnon \& S. Silverman (eds.), Complexities. Beyond Nature and Nurture, Chicago/Londres, The University of Chicago Press.

GLICK SCHILLER, Nina. (2007), "Beyond the Nation-State and its units of analysis: towards a new research agenda for migration studies". Essentials of Migration Theory, Working Paper 33, Bielefeld, COMCAD.

GOLDRING, Luin. (2002), "The Mexican State and transmigrant organizations: negotiating the boundaries of membership and participation". Latin American Research Review, 37 (3): 55-99.

GUARNIZO, Luis Eduardo \& SMITH, Michael Peter. (1998), "The locations of transnationalism”, in M. P. Smith y L. E. Guarnizo (eds.), Transnationalism from Below - Journal of Comparative Urban and Community Research, 6: 3-34.

JONES-CORREA, Michael. (2001), "Under two flags: dual nationality in Latin America and its consequences for naturalization in the United States". The International Migration Review, 35 (4): 997-1029.

JOPPKE, Christian. (2008), "Comparative citizenship: a restrictive turn in Europe?" Law \& Ethics of Human Rights, 2: 128-168.

KALM, Sara. (2008), Governing global migration. Lund Political Studies, No 153, Lund, Department of Political Science, Lund University.

KARASIK, Gabriela. (2000), “Tras la genealogía del diablo: discusiones sobre la nación y el Estado en la frontera argentino-boliviana”, in A. Grimson (comp.), Fronteras, naciones e identidades: la periferia como centro, Buenos Aires, Ciccus-La Crujía.

KARASIK, Gabriela. (2005), Etnicidad, cultura y clases sociales: procesos de formación histórica 
de la conciencia colectiva en Jujuy, 1970-2003. Tesis de doctorado. Universidad Nacional de Tucumán, Tucumán.

KIVISTO, Peter \& FAIST, Thomas. (2007), Citizenship: discourse, theory, and transnational prospects. Malden, Blackwell.

LAFLEUR, Jean-Michel (ed.). (2012), Diáspora y voto en el exterior: la participación politica de los emigrantes bolivianos en las elecciones de su pais de origen. Barcelona, Cidob.

LAGOS, Marcelo \& GUTIÉRREZ, Mirta. (2009), "La década del menemismo y la ingobernabilidad en Jujuy: nación, región y provincia en los noventa”, in M. Lagos (dir.), Jujuy bajo el signo neoliberal: política, sociedad y cultura en la década del noventa, San Salvador de Jujuy, EdiUnju.

LEVITT, Peggy \& GLICK SCHILLER, Nina. (2004), "Perspectivas internacionales sobre migración: conceptualizar la simultaneidad". Migración y Desarrollo, 3: 60-91.

LIEBERT, Ulrike. (2005), "What it means to be(come) a transatlantic citizen: rethinking postnational citizenship". New German Critique, 95: 93-105.

LOS TIEMPOS. (2009), "Gobierno cree que España malinterpretó la CPE”, 9 de julio de 2009. Disponible en: http://www.lostiempos. com/diario/actualidad/nacional/20090709/ gobierno-cree-que-espana-malinterpreto-la-cpe_24411_38190.html Consultado en: 20/10/2015.

MALLIMACI, Ana Inés. (2014), “Migraciones y género: las formas de la visibilidad femenina”, in M. J. Magliano y A. I. Mallimaci (comps.), Las mujeres latinoamericanas y sus migraciones, Villa María, Eduvim.

MANSUR DIAS, Guilherme. (2014), "Migraçao, segurança e governabilidade migratória: o papel dos organismos internacionais". Crítica y Emancipación: Revista Latinoamericana de Ciencias Sociales, Año VI, 11: 557-579.

MATEOS, Pablo \& DURAND, Jorge. (2012), "Residence vs. ancestry in acquisition of Spanish citizenship: a netnography approach". $\mathrm{Mi}$ graciones Internacionales, 6 (4): 9-46.

MINISTERIO de Justicia de Eespaña. (2012), Boletín del Ministerio de Justicia. Resoluciones de la
Dirección General de los Registros y del Notariado, Madrid, Secretaría General Técnica. Centro de Publicaciones.

MRE \& OIM (Ministerio de Relaciones Exteriores de Bolivia \& Organización Internacional para las Migraciones). (2011), Bolivianos y bolivianas en el exterior: propuestas de politica pública y ley para las migraciones, La Paz, OIM Bolivia. NCPE - Nueva Constitución Política del Estado. República de Bolivia, Asamblea Constituyente, Honorable Congreso Nacional. Texto final compatibilizado, octubre de 2008.

PEDONE, Claudia \& GIL ARAÚJO, Sandra. (2008), "Los laberintos de la ciudadanía: políticas migratorias e inserción de las familias emigrantes latinoamericanas en España”. REMHU - Revista Interdisciplinar da Mobilidade Humana, año XVI, 31: 143-164.

SALA, Gabriela. (2000), "Mano de obra boliviana en el tabaco y la cańa de azúcar en Jujuy, Argentina". Estudios Migratorios Latinoamericanos, 45: 337-370.

SASSEN, Saskia. (2000), "The need to distinguish denationalized and postnational”. Indiana Journal of Global Legal Studies, 7 (2): 575-582. SASSEN, Saskia. (2002), "The repositioning of citizenship: emergent subjects and spaces for politics". Berkeley Journal of Sociology, 46: 4-25.

$\mathrm{SDH}-\mathrm{MDH}$ (Secretaría de Derechos Humanos - Ministerio de Justicia y Derechos Humanos de la Nación Argentina). (2007), La Protección de Derechos de los Niños, Niñas y Adolescentes frente a la Explotación Laboral, Sexual, la Trata, el Tráfico y la Venta, Buenos Aires.

SHACHAR, Ayelet. (2009), The birthright lottery. citizenship and global inequality. Cambridge, Harvard University Press.

SIKKINK, Kathryn. (2003), "La dimensión transnacional de los movimientos sociales", in E. Jelin (comp.), Más allá de la nación: las escalas múltiples de los movimientos sociales, Buenos Aires, Libros del Zorzal.

SMITH, Michael Peter. (2007), "The two faces of transnational citizenship". Ethnic and Racial Studies, 30 (6): 1096-1116.

SMITH, Michael Peter \& GUARNIZO, Luis Eduardo.(2009), "Global mobility, shifting 
borders and urban citizenship".Tijdschrift voor Economische en Sociale Geografie, 100c(5): 610622.

SMITH, Michael Peter \& BAKKER, Matt.(2008), Citizenship across borders: the political transnationalism of El Migrante. Ithaca/London, Cornell University Press.

SMITH, Robert. (2003), "Migrant membership as an instituted process: transnationalization, the State and the extra-territorial conduct of Mexican politics". The International Migration Review, 37 (2): 297-343.

SOLOMON, M. Scott. (2009), "State-led migration, democratic legitimacy, and deterritorialization: the Philippines' labour export model". European Journal of East Asian Studies, 8 (2): 275-300.

SOYSAL, Yasemin. (1994), Limits of citizenship: migrants and postnational membership in Euro$p e$. Chicago, The University of Chicago Press.

STOLCKE, Verena (1992), Racismo y sexismo en la Cuba colonial. Madrid, Alianza Editorial.

STOLCKE, Verena (1999), "La nueva retórica de la exclusión en Europa". International Social Science Journal - ISSJ, LI (159). Disponible en http://www.unesco.org/issj/rics159/stolckespa. html, consultado en 21/08/2008.

STOLCKE, Verena. (2000), "La 'naturaleza' de la nacionalidad". Desarrollo Económico, 40 (157): 23-43.

TORPEY, John. (2000), The invention of the passport: surveillance, Citizenship and the State. Cambridge, Cambridge University Press.

TRUCCO, Marcelo. (2007), "La nacionalidad en el derecho internacional: régimen jurídico de la nacionalidad argentina". Infojus - Sistema Argentino de Información Jurídica. Disponible en http://www.infojus.gob.ar/doctrina/dasf070028-trucco-nacionalidad_en_derecho_internacional.htm, consultado en 16/10/2015.

VAN DONGEN, Els. (2017), "Behind the ties that bind: diaspora-making and Nation-building in China and India in historical perspective, 1850s-2010s". Asian Studies Review, 41(1): 117-135.

VERTOVEC, Steven. (2006), "Transnacionalismo migrante y modos de transformación", in A.
Portes \& J. de Wind (coords.), Repensando las migraciones: nuevas perspectivas teóricas y empiricas, México, Secretaría de Gobernación Instituto Nacional de Migración.

VVAA. (2010), Los niños y las niñas no estamos en venta. Pastoral Migratoria - Prelatura de Humahuaca.

WALTERS, William. (2015), "Reflections on migration and governmentality". Movements Journal fürkritische Migrations - und Grenz regime forschung, 1 (1): 1-30. 


\section{CONJURAR O ACIDENTE DE NASCER: MIGRAÇĀO, POPULAÇÃO, NACIONALIDADE E CIDADANIA}

\section{Sergio Caggiano}

Palavras-chaves: Migrações; Nacionalidade e cidadania; Estados.

A atribuição de nacionalidade é um dos principais dispositivos estatais para a codificação e regulação da mobilidade humana. É uma parte substantiva da produção de população (Foucault) que os Estados realizam em processos dinâmicos e conflituosos, estruturados por valores de classe e gênero. $O$ artigo analisa as açôes de quatro Estados em duas situaçôes envolvendo migrantes da Bolívia na Argentina e na Espanha. A concessão ou não da nacionalidade aos imigrantes e emigrantes, e aos seus filhos, causa impacto nas práticas cidadâs e no exercício de direitos dos cidadãos atuais e futuros. Assim como alguns autores apontam que existem formas de emancipação da cidadania em relação à nacionalidade, a principal contribuição deste artigo é expor, no sentido inverso, intervençóes que reforçam esse vínculo. Os dados empíricos são produto de trabalho de campo etnográfico multissituado e de revisão de fontes secundárias e documentos institucionais entre 2005 e 2015.

\section{TO CONJURE THE ACCIDENT OF BEING BORN: MIGRATION, POPULATION, NATIONALITY AND CITIZENSHIP}

\author{
Sergio Caggiano
}

Keywords: Migrations, Nationality and Citizenship, States.

The granting of nationality is one of the principal state devices to codify and regulate human mobility. It is a key element of the state production of population (Foucault), which is a dynamic and conflictive process, structured by class and gender values. This article explores the actions of four States in two situations, involving migrants from Bolivia in Argentina and Spain. The granting or not of nationality to immigrants and emigrants, and their offspring, impacts on citizenship practices and the enforcement of rights of current and future citizens. In contrast to what some authors have pointed out as forms of emancipation of citizenship in relation to nationality, the main contribution of this article is to expose interventions that reinforce the link between them. The empirical data result from multisite ethnographic fieldwork and review of secondary sources and institutional documents between 2005 and 2015.

\section{CONJURER L'ACCIDENT DE NAÎTRE : MIGRATION, POPULATION, NATIONALITÉ ET CITOYENNETÉ}

\author{
Sergio Caggiano
}

Mots-clés: Migrations; Nationalité et citoyenneté; États.

L'attribution de la nationalité est l'un des principaux dispositifs publics de codification et de régulation de la mobilité humaine. C'est une partie importante de la production de population (Foucault) que les États exécutent dans des processus dynamiques et conflictuels, structurés par des valeurs de classe et de genre. L'article analyse les actions de quatre États dans deux situations impliquant des migrants de Bolivie en Argentine et en Espagne. L'octroi ou pas de la nationalité aux immigrants et aux émigrants, ainsi qu'à leurs enfants, a un impact sur les pratiques des citoyens et sur l'exercice des droits des citoyens actuels et futurs. Tout comme certains auteurs soulignent qu'il existe des formes d'émancipation de la citoyenneté par rapport à la nationalité, la principale contribution de cet article est d'exposer, dans la direction opposée, des interventions qui renforcent ce lien. Les données empiriques sont un produit de travail ethnographique polyvalent sur le terrain et l'examen de sources secondaires et de documents institutionnels entre 2005 et 2015 . 


\section{CONJURAR EL ACCIDENTE DE NACER: MIGRACIÓN, POBLACIÓN, NACIONALIDAD Y CIUDADANÍA}

\section{Sergio Caggiano}

Palabras clave: Migraciones; Nacionalidad y ciudadanía; Estados

La atribución de nacionalidad es un dispositivo estatal clave para codificar y regular la movilidad humana. Es parte sustantiva de la producción de población (Foucault) que los Estados llevan adelante en procesos dinámicos y conflictivos, estructurados por valores de clase y género. El artículo revisa acciones de cuatro Estados en dos situaciones que involucran migrantes procedentes de Bolivia en Argentina y en Espańa. El otorgamiento o no de nacionalidad a in- y emigrantes y a sus hijos impacta en las prácticas ciudadanas y el ejercicio de derechos de actuales y de futuros ciudadanos. Así, de cara a lo que algunos autores señalan como formas de emancipación de la ciudadanía respecto de la nacionalidad, el principal aporte del artículo es exponer, en dirección inversa, intervenciones que refuerzan este vínculo. Los datos empíricos resultan de un trabajo de campo etnográfico multisituado y de revisión de fuentes secundarias y documentos institucionales entre 2005 y 2015. 